ojs.uv.es/index.php/qfilologia/index

Rebut: 16.06.2020. Acceptat: 23.08.2020

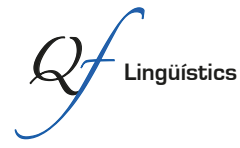

Per a citar aquest article: García Benito, Ana Belén. 2020. “Fraseodidáctica. Marcos situacionales para trabajar los Enunciados Fraseológicos en la clase de PLE para hispanohablantes”. Quaderns de Filologia: Estudis Lingüístics XXV: I35-I49.

doi: $10.7203 /$ Q.25.19076

\title{
Fraseodidáctica. Marcos situacionales para trabajar los Enunciados Fraseológicos en la clase de PLE para hispanohablantes
}

\author{
Phraseodidactic. Situational frameworks for working on Phraseological Statements \\ in the PLE class for Spanish speakers
}

\author{
Ana Belén García Benito \\ Universidad de Extremadura \\ agbenito@unex.es
}

Resumen: El objetivo de este trabajo es delimitar las funciones pragmático-discursivas de algunos de los Enunciados Fraseológicos más habituales del portugués, ofreciendo los "marcos situacionales" (Corpas Pastor, I996) que harán posible su comprensión y funcionamiento por parte de estudiantes hispanohablantes. Los "marcos situacionales" que ofrecemos los encuadran en la interacción dinámica existente entre hablante y oyente, proporcionando toda la información necesaria para su uso: características de los participantes en la interacción, características del escenario, su motivación, el contexto -secuenciación, elementos prosódicos, lenguaje no verbal, registro-, variantes, sinónimos, así como los conocimientos socioculturales compartidos por los hablantes.

Palabras clave: fraseología portuguesa; enunciados fraseológicos; pragmática; marcos situacionales; portugués lengua extranjera.

Abstract: The objective of this work is to delimit the pragmatic-discursive functions of some of the most common Phraseological Statements in Portuguese, offering the "situational frames" (Corpas Pastor, I996) that will make it possible for Spanish-speaking students to understand and function. The "situational frames" we offer are framed in the dynamic interaction between speaker and listener, providing all the necessary information for their use: characteristics of the participants in the interaction, characteristics of the scenario, their motivation, the context -sequence, prosodic elements, non-verbal language, register-, variants, synonyms, as well as the socio-cultural knowledge shared by the speakers.

Keywords: Portuguese phraseology; phraseological statements; pragmatics; situational frams; Portuguese foreign language. 


\section{Introducción}

El objetivo de nuestro estudio es establecer patrones productivos de análisis que faciliten el aprendizaje de Enunciados Fraseológicos (EFS) a estudiantes hispanohablantes de Portugués Lengua Extranjera (PLE), conscientes de que se trata de uno de los tipos más complejos de Unidades Fraseológicas (UFS) y de las dificultades para trabajarlos en el aula.

Asumiendo el "giro pragmático" propuesto por Olza \& Manero (2013), cada vez son más numerosos los trabajos que realizan una aproximación pragmático-discursiva a la Fraseología, con el objetivo de proporcionar explicaciones satisfactorias sobre el comportamiento real, la productividad y la manera en que los hablantes utilizan las UFS. Es necesario, en la actualidad, complementar trabajos anteriores centrados en consolidar aspectos semánticos, formales o lexicográficos, con otros que analicen el funcionamiento de la Fraseología en su uso discursivo o conversacional real. La necesidad de encarar la Pragmática como perspectiva de análisis lingüístico que complemente las conclusiones obtenidas desde otros enfoques, parece hoy clara en los estudios fraseológicos.

\section{Marcos situacionales: estructura conceptual para trabajar Fraseología}

En función de la perspectiva pragmática asumida, resulta evidente la necesidad de desarrollar patrones productivos de análisis de las UFS, especialmente necesarios, a nuestro juicio, en el caso de los EFS, al enunciarse en unas circunstancias concretas, por lo que su interpretación y uso están determinados por el contexto lingüístico y situacional. La dependencia situacional de los EFS constituye, sin duda, uno de los aspectos más significativos de estos fraseologismos, de ahí que para utilizarlos, comprender e interpretar el conocimiento que encierran, sea imprescindible "tener en cuenta los aspectos socioculturales de la comunidad correspondiente, así como las situaciones que propician el uso" (Corpas Pastor, I996: I76).

Ante esta necesidad, Coulmas (I979) propone delimitar las situaciones que dan lugar al uso de los fraseologismos, dando cuenta de ellas en los denominados situacional frames. Para este autor, los hablantes conciben determinados elementos de la Fraseología como formando parte de marcos concretos. Se trata de estructuras conceptuales que contienen información sobre objetos 
y situaciones estereotipadas que los hablantes asocian a esas unidades, así como toda la información sobre la secuenciación y partes que constituyen la situación o los roles semánticos que desempeñan los participantes. De esta manera, para Coulmas (I979: 244) los situacional frames son:

Esquemas cognitivos que a) comprenden la información necesaria para el uso correcto de una fórmula o una clase de fórmulas y b) motivan el uso de la(s) misma(s) en un momento dado. Dichas estructuras de conocimiento representan la percepción prototípica y convencional de tales situaciones por parte de los miembros de la comunidad hablante, siendo fiel reflejo de su cultura.

Posteriormente, otros autores como Ameka (1987: 302) proponen alternativas diversas con el propósito idéntico de ofrecer una caracterización lo más completa posible de la situación que motiva el fraseologismo:

- Especificación rigurosa del conjunto de condiciones de cada fórmula.

- Semántica de los prototipos.

- Descomposición en primitivos semánticos independientemente de la cultura que se trate.

Sin embargo, será Corpas Pastor quien, en su conocido Manual de fraseología española, lleve a la práctica estas teóricas, ilustrando la codificación social y cultural presente en EFS rutinarios como jA la orden!, así como las situaciones que lo provocan. Establece, por tanto, el marco de situación -traducción literal de los situacional frames de Coulmas-, con información sobre los participantes en la situación de uso de ese enunciado, el escenario, la motivación, las restricciones contextuales y las acciones concomitantes asociadas -gestualidad- (Corpas Pastor, I996: I77).

\section{Enunciados fraseológicos del portugués y del español}

En la Fraseología, los EFS integran el grupo constituido por las secuencias autónomas de habla. Son también las unidades que muestran una mayor frecuencia de uso en el discurso, especialmente en el coloquial (Solano Rodríguez, 20I7).

En nuestra aproximación a estos fraseologismos seguiremos la formulación de Solano Rodríguez (20I2), que parte de la siguiente clasificación de la Fraseología: 


\begin{tabular}{|c|c|c|c|}
\hline Disciplina & Ámbito & Tipos & Subtipos \\
\hline \multirow{8}{*}{ Fraseología } & \multirow{7}{*}{ Fraseología } & \multirow{2}{*}{ Sintagmas Fraseológicos } & Colocaciones \\
\hline & & & Locuciones \\
\hline & & \multirow{4}{*}{ Enunciados fraseológicos } & E. F. rutinarios \\
\hline & & & E. F. pragmáticos \\
\hline & & & E. F. discursivos \\
\hline & & & E. F. proverbiales \\
\hline & & Esquemas sintácticos & - \\
\hline & Paremiología & Paremias & $\begin{array}{l}\text { Refranes, proverbios, frases } \\
\text { proverbiales, dialogismos, } \\
\text { adagios, apotegmas, } \\
\text { máximas. }\end{array}$ \\
\hline
\end{tabular}

Figura I. Clasificación de la Fraseología

Y definimos nuestro objeto de estudio -los enunciados fraseológicos- partiendo también de los presupuestos de esta autora (Solano Rodríguez, 20I2: I27), como:

- Actos de habla que no necesitan integrarse con otros signos lingüísticos para su funcionamiento en el discurso.

- Ilocutivos o perlocutivos: de uso en la conversación preferentemente.

- Con independencia enunciativa.

- Con significado pragmático-situacional.

- Facilitadores de la interacción.

Distinguiendo cuatro tipos:

I. Rutinarios: convenciones psicosociales que regulan una buena interacción As melhoras! // ¡Que te mejores!

2. Pragmáticos: modalizadores del discurso Era só o que faltava! // ¡Lo que nos faltaba!

3. Discursivos: fundamentales para ordenar o realzar el discurso del hablante en multitud de funciones expresivas y asertivas Resumindo e concluindo // Em resumidas cuentas.

4. Proverbiales: metáforas lexicalizadas con valor paremiológico pero no referencial Por falar no Mendes, à porta o tendes // Hablando del rey de Roma, por la puerta asoma. 
Características definitorias como la polilexicalidad, la fijación, la institucionalización y la idiomaticidad son comunes a los EFS y al resto de unidades de la Fraseología. En el caso de la fijación, no obstante, en los EFS, además de fijación formal, distinguimos también fijación semántico-pragmática (Solano Rodríguez, 2007), al presentar un significado fijado por el uso que se les da en el contexto habitual en el que se producen. Olha o passarinho! // iMira al parajito! presenta fijación formal y semántico-pragmática, ya que su significado solamente puede aparecer en la situación concreta en la que alguien está haciendo una fotografía y advierte a su(s) interlocutor(es) de que debe mirar en la dirección en la que está situada la cámara. De igual manera, creemos que debe ser considerada la idiomaticidad pragmática de los EFS, puesto que además de su mayor o menor grado de opacidad semántica, muchos presentan una unión tan elevada con el contexto pragmático, que su interpretación y uso implican conocer la situación que propicia su utilización. Así, el significado final de un enunciado como Estamos conversados! // ¡No se hable más! depende de la relación que se establece entre sus constituyentes léxicos y el siguiente contexto situacional: inmediatamente después de una intervención propia o del interlocutor, sobre la que se considera que no es necesario continuar hablando.

Rasgos a los que debemos añadir los siguientes, específicos de los EFS:

I. Independencia: Alvarado Ortega (20I5) establece diversos tipos de independencia en los EFS. Independencia sintáctica: pues constituyen actos de habla por sí mismos. Independencia semántica: significan por ellos mismos, en función del contexto habitual en el que se producen. Independencia textual: autonomía respecto al contexto lingüístico. Los EFS se distinguen de las Paremias por carecer de independencia textual, pues a diferencia de estas, dependen de las situaciones comunicativas concretan en las que se formulan. Independencia distribucional: pueden aparecer en el discurso tantas veces como sea necesario, pues es el contexto situacional y la actitud del hablante ante un hecho lo que provocará su aparición. Independencia entonativa: son actos de habla que suelen tener un esquema entonativo propio.

2. Significado pragmático-situacional: los EFS se enuncian en unas circunstancias concretas, de ahí que tengan un significado más pragmático que semántico-lexical. Por falar no Mendes, à porta o tendes // Hablando del rey de Roma, por la puerta asoma, adquiere su significado total solamente en la situación concreta en la que alguien está hablando sobre una persona y esa persona aparece en ese preciso momento. 
3. Intencionalidad: buena parte de ellos se dicen directamente al interlocutor, para influir en su conducta És de Braga? // iQue se escapa el gato! -con la intención de que alguien cierre una puerta que ha dejado abierta-, o a modo de comentario que se quiere dar a conocer al interlocutor Pariu a galega! // ¡Éramos pocos y parió la abuela!

4. Actos ilocutivos/perlocutivos: como refuerzo o respuesta de actos ilocutivos Não tem de que // No hay de qué.

5. Expresividad: los EFS rutinarios presentan el grado cero de expresividad, ya que son convencionales Se faz favor // Por favor. Los proverbiales contienen un nivel medio de expresividad Cada macaco ao seu galho // Cada mochuelo a su olivo. Los discursivos, en cambio, poseen expresividad en grado elevado, desempeñando funciones como controlar que el interlocutor comprende lo que estamos diciendo Como podes calcular // Como puedes imaginarte; manifestarnos a cerca de lo que nuestro interlocutor ha dicho Esta-se mesmo a ver! // iEso habrá que verlo!; tomar el turno Por falar nisso // Ahora que lo dices. Finalmente, en los pragmáticos el grado de expresividad es tan elevado que pueden actuar como modalizadores del discurso, intensificando, matizando, etc., lo expresado por el interlocutor Quando as galinhas tiverem dentes! // ¡Cuando las ranas críen pelo!

6. Connotaciones histórico-culturales: los EFS reflejan en mayor o menor medida la cultura que ha determinado su aparición. De entre ellos, los proverbiales son los que con más frecuencia se remontan a episodios históricos, por lo que Solano Rodríguez (20I2: I24) los considera verdaderos fósiles lingüísticos: Tudo como dantes, quartel general em Abrantes, Alma até Almeida.

\section{Marcos situacionales para trabajar EFS del portugués con hispanohablantes}

Nuestro objetivo en este apartado no es otro que delimitar las funciones pragmático-discursivas de algunos de los EFS más habituales del portugués ${ }^{\mathrm{I}}$, ofreciendo los marcos situacionales que harán posible su comprensión y facilitarán su utilización por parte de estudiantes hispanohablantes. Para ello, partimos del modelo de marco situacional ofrecido por Corpas Pastor (I996), al que hemos realizado algunas modificaciones.

\footnotetext{
${ }^{\mathrm{I}}$ Nos centramos en este trabajo en el portugués europeo, dejando fuera del ámbito de estudio el portugués de Brasil.
} 
Dado que, para poder comprender y explicar la confluencia en el discurso de los EFS, no es suficiente el contexto lingüístico, entendemos que el marco situacional debe contener todos los elementos que hacen posible el uso del enunciado y lo motivan en un determinado momento. Los marcos situacionales, por lo tanto, deben encuadrar los EFS en la interacción y en la relación dinámica existente entre hablante y oyente, proporcionando toda la información necesaria para su uso.

Así, deberán contener, en primer lugar, información sobre los participantes en la situación, con datos sobre el sexo, la edad, el papel social o el grado de familiaridad existente entre los hablantes. Para que los estudiantes de portugués puedan llegar a utilizar correctamente un determinado enunciado, tendrán que conocer si este se utiliza independientemente o no del sexo de los interlocutores, si estos son o no personas adultas, si uno de ellos posee o no algún grado de autoridad sobre el otro, o la familiaridad que existe entre ellos.

El segundo bloque de informaciones que contienen nuestros marcos situacionales está relacionado con el escenario, con datos sobre el tiempo y el lugar, pues los estudiantes necesitan conocer si los interlocutores deben estar próximos o no a la hora de utilizar el enunciado, si pueden usarlo en interior o exterior, etc.

Fundamental también resulta el tercer apartado presente en los marcos de situación que proponemos: la motivación. Se trata de la razón que mueve al hablante A a utilizar determinado enunciado en su intercambio conversacional con B. En el caso del ejemplo de Corpas Pastor jA la orden!, la motivación parece clara: el hablante A -inferior jerárquico- expresa al hablante B -su superior- que el canal de comunicación está abierto, que lo reconoce como superior y espera órdenes o información.

El siguiente apartado, denominado contexto $^{2}$, resulta de la fusión de los apartados "restricciones contextuales" y "acciones concomitantes" propuestos por Corpas Pastor (1996). En él se ofrece información sobre la secuenciación habitual de utilización del enunciado en la interacción hablante-oyente. A este respecto, Luque Nadal (2008: 89) habla de dos tipos de situaciones: las situaciones prácticas y las situaciones lingüísticas. Las primeras dependen de algo que ocurre o bien de lo que alguien ha hecho o está haciendo. Por ejemplo, el que alguien haya dejado la puerta abierta al entrar es la razón por la cual otra

\footnotetext{
${ }^{2}$ Buena parte de la información contenida en este apartado procede de las "Fichas" que acompañan a las diversas entradas del Refranero Multilingüe del Centro Virtual Cervantes https:// cvc.cervantes.es/lengua/refranero/
} 
persona puede utilizar el enunciado És de Braga? Las segundas, por el contrario, son una reacción ante algo que alguien ha dicho. Así, utilizamos un enunciado como Não tem de que! inmediatamente después de que alguien nos dé las gracias. El contexto contiene también información sobre el registro de uso del enunciado -coloquial, neutro...- , un marcador de uso con indicaciones referentes a la frecuencia de uso en la lengua hablada contemporánea -muy usado, poco usado, en desuso-, información sobre los elementos prosódicos paralingüísticos -entonación, nivel de expresividad- o sobre el lenguaje no verbal -gestos, mímica-, que acompañan al enunciado. Proporciona asimismo información sobre posibles variantes del enunciado, además de EFS sinónimos -si los tuviera- que podrían utilizarse en situaciones idénticas.

Completa nuestros marcos el apartado conocimientos socioculturales, con información socio-cultural asociada al enunciado: su posible origen, por ejemplo, o acontecimientos históricos con él relacionados.

Finalmente, dado que buena parte de las situaciones de uso de los EFS implican intercambios conversacionales o bien aparecen en textos escritos que reproducen dichos intercambios, hemos considerado conveniente completar nuestros marcos situacionales con un pequeño diálogo creado ad hoc para contextualizar el fraseologismo3.

Ofrecemos, a continuación, a modo de ejemplo, los marcos situacionales de algunos EFS del portugués, acompañados de las realizaciones lingüísticas equivalentes en español, asumiendo de esta forma una metodología compara$\mathrm{da}$, teniendo en cuenta que orientamos nuestro trabajo a estudiantes hispanohablantes. Dichas realizaciones, EFS también, irán acompañadas igualmente de sus correspondientes marcos situacionales. Partimos para ello de la base de que portugués y español comparten un buen número de situaciones. En este sentido, Quiroga (2004) habla de una estructura conceptual -tal vez metafórica- compartida por varias culturas y que proporciona fraseologismos semejantes. De esta forma, las equivalencias en español que proponemos para los EFS del portugués deben ser consideradas equivalentes pragmáticos; en modo alguno traducciones literales. No importa si la metáfora que se utiliza en ambas lenguas o la manera de expresar una idea no es la misma, lo esencial es que el

\footnotetext{
${ }^{3}$ Futuros repertorios de marcos situacionales del portugués y del español requerirán la inclusión de textos auténticos, si bien en este caso, al tratarse de una primera aproximación, hemos optado por diálogos fabricados.

4 Somos conscientes del escaso número de ejemplos que ilustran nuestra propuesta. Sin embargo, por motivos de espacio resulta imposible incluir un corpus más amplio que contribuya a argumentar con más contundencia la propuesta de trabajo.
} 
fraseologismo se utilice en el mismo contexto situacional y su uso provoque el mismo efecto. No obstante, no debemos olvidar que hay EFS para los cuales no encontraremos equivalentes pragmáticos en otras lenguas, pues son un reflejo de la cultura que les dio origen. Especialmente cuando la situación de uso de un fraseologismo en una determinada lengua no existe como tal en otra lengua.

\begin{tabular}{|l}
\hline \multicolumn{1}{|c|}{ Pariu a galega? } \\
\hline PARTICIPANTES \\
Sexo: se puede utilizar independientemente del sexo de los interlocutores. \\
Edad: los interlocutores son personas adultas. \\
Familiaridad: alto grado de familiaridad entre los interlocutores. \\
\hline ESCENARIO \\
Tiempo: no hay restricciones temporales. \\
Lugar: no hay restricciones de lugar. \\
\hline MOTIVACIÓN \\
Razón: el hablante A lo utiliza para manifestar a B su sorpresa o extrañeza al haber mu- \\
chas personas en un determinado lugar.
\end{tabular}

\section{RESTRICCIONES CONTEXTUALES}

Secuenciación: el hablante A lo utiliza cuando encuentra muchas personas en un lugar en el que no esperaba tantas o tanta confusión.

Elementos prosódicos: se pronuncia con un esquema de entonación propio: curva ascendente; alto nivel de expresividad: asombro, extrañeza por parte de hablante.

Registro: coloquial; muy informal.

Marcador de uso: poco usado.

Variantes: Pariu aqui a galega?; Parece que pariu aqui a galega!; Aqui pariu a galega!

Sinónimos: Éramos trinta, pariú nossa avó.

\section{CONOCIMIENTOS SOCIOCULTURALES}

A finales del S. XIX y principios del X, la imagen de Galicia y de los gallegos en Portugal ofrece claramente un imagotipo negativo que responde al fenómeno migratorio gallego en Portugal, fundamentalmente en Lisboa. La posición/función social ejercida por los gallegos en Portugal hasta mediados del S. XX alimenta el imaginario negativo. De esta manera, los gallegos son considerados groseros, brutos, ignorantes, avaros, trabajadores no cualificados, en ocasiones también alcohólicos, ingenuos pero desconfiados, utilizadores de una variedad lingüística propia y de una vestimenta peculiar. La vitalidad de este imagotipo negativo quedó patente en numerosos productos culturales (literatura, cine, etc.), así como en la lengua, especialmente en la Fraseología. De manera paralela y a medida que avanza el S. XX, este imagotipo negativo comienza a convivir con una imagen de afinidad respecto a los gallegos. En la actualidad, fraseologismos como Pariu a galega? están desprovistos de función humorística y carga despectiva en el espacio social portugués, ya que el fenómeno migratorio dejó de tener continuidad a mediados del S. XX.

[Pazos, Carlos (2012), "Galegos, galego-portugueses ou espanhóis? Hipóteses e contributos para a análise das origens e funções da imagem atual da Galiza e dos galegos en Portugal", Diacrítica, vol. 26, n² 2, pp. 431-443.] 


\section{DIÁLOGO}

A- Pariu a galega? Tantas pessoas!! Meu Deus! E continuam a chegar!

B- Pois é. Temos um grande problema, sim senhor.

A- A sala tem capacidade para cinquenta pessoas...

$\mathrm{B}-$... e aqui deve haver umas duzentas!

A- Talvez mais...

Figura 2. Marco situacional Pariu a galega?

\section{¡Éramos pocos $y$ parió la abuela!}

\section{PARTICIPANTES}

Sexo: se puede utilizar independientemente del sexo de los interlocutores.

Edad: los interlocutores son personas adultas.

Familiaridad: alto grado de familiaridad entre los interlocutores.

ESCENARIO

Tiempo: no hay restricciones temporales.

Lugar: no hay restricciones de lugar.

\section{MOTIVACIÓN}

Razón: el hablante A lo utiliza para manifestar a B su sorpresa o extrañeza al haber muchas personas en un determinado lugar.

\section{RESTRICCIONES CONTEXTUALES}

Secuenciación: el hablante A lo utiliza cuando encuentra muchas personas en un lugar en el que no esperaba tantas o tanta confusión.

Elementos prosódicos: se pronuncia con un esquema de entonación propio: curva descendente; alto nivel de expresividad: asombro, extrañeza por parte de hablante.

Registro: coloquial.

Marcador de uso: poco usado.

Variantes: ¡Éramos pocos, $y$ parió la burra”; ¡Éramos pocos y parió la gata!;jÉramos compañuela, y parió nuestra abuela!; jÉramos treinta, y parió nuestra abuela!

Sinónimos: ¡No cabemos al fuego y parió mi suegra!; No cabíamos al fuego, y entró nuestro abuelo!; ¡Pongo un circo, y me crecen los enanos!

\section{CONOCIMIENTOS SOCIOCULTURALES}

El origen del fraseologismo pudiera residir en el siguiente hecho histórico: el rey Alfonso VIII de Castilla contrajo matrimonio con Leonor de Inglaterra en 1170, de cuya unión nacieron diez hijos. El rey Alfonso, es recordado por su importante papel en la Reconquista -consiguió una tregua con los musulmanes que duró hasta 1210-, así como por haber puesto fin al conflicto con el Reino de León, gracias al matrimonio de su primogénita, Doña Berenguela, con el rey Alfonso IX de León en 1197. Este matrimonio duró hasta que el Papa Inocencio III lo disolvió en 1204 por razones de consanguineidad. Doña Berenguela regresó entonces con sus hijos a la corte de sus padres en Castilla y ese mismo año su madre dio a luz a su último hijo, Enrique, a los 44 años.

https://spanish.stackexchange.com/questions/27726/cu\%C3\%All-es-el-origen-del-dicho\% $3 \% 8$ gramos-pocos-y-pari\%C3\%B3-la-abuela 


\section{DIÁLOGO}

A- ¡Éramos pocos y parió la abuela! ¡Qué cantidad de gente! !Dios! ¡Y no dejan de llegar!

B- Pues sí. Tenemos un problema serio. Sí señor.

A- Esta sala tiene capacidad para cincuenta personas más o menos...

B- ... jY aquí debe haber unas doscientas!

A- ¡O más!

Figura 3. Marco situacional ¡Éramos pocos y parió la abuela!

\begin{tabular}{|l|}
\hline \multicolumn{1}{|c|}{ O mundo é pequeno! } \\
\hline PARTICIPANTES \\
Sexo: se puede utilizar independientemente del sexo de los interlocutores. \\
Edad: los interlocutores son personas adultas. \\
Familiaridad: alto grado de familiaridad entre los interlocutores. \\
\hline ESCENARIO \\
Tiempo: no hay restricciones temporales. \\
Lugar: no hay restricciones de lugar. \\
\hline MOTIVACIÓN \\
Razón: el hablante A lo utiliza para hacer saber a B que no esperaba encontrarlo en ese \\
lugar. \\
\hline RESTRICCIONES CONTEXTUALES \\
Secuenciación: el hablante A lo utiliza cuando encuentra a B inesperadamente o en un \\
lugar insospechado. \\
Elementos prosódicos: se pronuncia con un esquema de entonación propio: curva des- \\
cendente; alto nivel de expresividad: sorpresa por parte de hablante. \\
Registro: neutro. \\
Marcador de uso: muy usado \\
Variantes: Como o mundo é pequeno! \\
Sinónimos: Não se pode andar escondido! \\
\hline DIÁLOGO \\
A- Ainda não acredito que estejamos no Rio de Janeiro! \\
B- Pois é. Depois de tanto tempo a desejar vir, conseguimos! Vamos é aproveitar o dia de praia! \\
A- Sim. Copacabana é linda de morrer... \\
B- Mas, espera aí. Essa que está no posto dos gelados não é a tua colega Mafalda? \\
A- É sim. O mundo é pequeno! Mafalda! Mafalda! \\
\hline
\end{tabular}

Figura 4. Marco situacional O mundo é pequeno! 


\section{¡El mundo es un pañuelo!}

\section{PARTICIPANTES}

Sexo: se puede utilizar independientemente del sexo de los interlocutores.

Edad: los interlocutores son personas adultas.

Familiaridad: alto grado de familiaridad entre los interlocutores.

\section{ESCENARIO}

Tiempo: no hay restricciones temporales.

Lugar: no hay restricciones de lugar.

\section{MOTIVACIÓN}

Razón: el hablante A lo utiliza para hacer saber a B que no esperaba encontrarlo en ese lugar.

\section{RESTRICCIONES CONTEXTUALES}

Secuenciación: el hablante A lo utiliza cuando encuentra a B inesperadamente o en un lugar insospechado.

Elementos prosódicos: se pronuncia con un esquema de entonación propio: curva descendente; alto nivel de expresividad: sorpresa por parte del hablante.

Registro: coloquial.

Marcador de uso: muy usado.

\section{CONOCIMIENTOS SOCIOCULTURALES}

Es probable que el origen de este fraseologismo proceda del hecho de que en latín "pañuelo" es mappa. En la Antigua Roma, el mappa era una especie de lienzo o pañuelo que se utilizaba para dar la señal de inicio de los juegos. Tras la caída del Imperio Romano, continuó utilizándose la palabra para referirse a los trozos de tela sobre los cuales se dibujaban los linderos de terrenos o propiedades. Con el tiempo, cualquier representación de una parte o de toda la superficie terrestre fue denominada mappa, como los mappae mundi medievales, literalmente 'lienzos sobre los que se dibuja el mundo'.

Diccionario Etimológico de Chile http://etimologias.dechile.net/

\section{DIÁLOGO}

A- ¡Todavía no me puedo creer que estemos en Río de Janeiro!

B- Es verdad. Después de tanto tiempo deseando venir iLo hemos conseguido! ¡Vamos a aprovechar este día de playa!

A- Sí. Copacabana es una maravilla...

B- Espera un momento. ¿Esa que está en el puesto de helados no es tu compañera Mafalda? A- ¡Pues claro que sí! ¡El mundo es un pañuelo! ¡Mafalda! ¡Mafalda!

Figura 5. Marco situacional ;El mundo es un pañuelo!

\section{Conclusiones}

Los EFS pueden ser considerados periféricos respecto al núcleo duro de la Fraseología, pero en modo alguno marginales, dada su elevada frecuencia de uso en el discurso, especialmente en el coloquial. Sin embargo, la enseñan- 
za-aprendizaje de LE continua desestimando el componente fraseológico en general y estos fraseologismos en particular.

En el caso concreto de los EFS del portugués, su alta incidencia en el discurso, unido a su escasa presencia tanto en las clases como en los materiales de trabajo, nos obligan a reclamar para este componente de la lengua el lugar que merece en la didáctica del PLE. Abogamos por un tratamiento sistemático y específico para las UFS a la hora de diseñar los contenidos curriculares de las LE, tal y como establece el Marco Común Europeo de Referencia para las Lenguas (MCERL) (2002), incluso desde el nivel Ai de aprendizaje. Solano Rodríguez (2007: 203) defiende que la competencia fraseológica forma parte de la competencia comunicativa junto con la competencia pragmática, sociolingüística y discursiva. De esta forma, si pretendemos que la lengua que adquieren nuestros estudiantes se aleje de un habla estereotipada y carente de expresividad, debemos incorporar el componente fraseológico. No obstante, conocer las unidades léxicas no es suficiente. Nuestros estudiantes deben ser competentes en el uso de las UFS, de ahí la necesidad de asumir una perspectiva pragmática que complete la aproximación a la Fraseología realizada desde otros enfoques.

Dentro de esta perspectiva pragmática, nuestra propuesta en este trabajo ha sido mostrar que definir los marcos situaciones para los EFS del portugués, puede ser un complemento al trabajo sintáctico, semántico, etc., con estos fraseologismos en la clase de PLE. Al presentar a nuestros estudiantes estos marcos, estaremos ofreciéndoles esquemas cognitivos que representan la percepción prototípica de una determinada situación por parte de los integrantes de una comunidad. Es decir, estaremos proporcionándoles toda la información sobre las situaciones concretas en las que se utilizan los EFS, lo cual estamos convencidos de que será positivo a la hora de reproducir todos los matices semánticos, expresivos y connotativos de los EFS. Al mismo tiempo, los profesores de PLE encontrarán en ellos la base para elaborar actividades con las que trabajarlos en sus clases.

\section{Bibliografía}

Ameka, Felix. 1987. A comparative analysis of linguistic routines in two languages, English and Ewe. Journal of Pragmatics II: 299-326. doi: https://doi. org/10.10I6/0378-2166(87)90135-4

Alvarado Ortega, M. ${ }^{a}$ Belén. 20I5. Independencia y fórmulas rutinarias. Reestructuración de la Esfera III. Revista Española de Lingüística Aplicada 28(I): I-I6. 
Consejo de Europa. 2002. Marco común europeo de referencia para las lenguas: aprendizaje, enseñanza, evaluación. Alcalá de Henares: Instituto Cervantes.

Corpas Pastor, Gloria. I996. Manual de fraseología española. Madrid: Gredos.

Corpas Pastor, Gloria \& Morvay, Károly. 2002. Los estudios de fraseología y fraseografía en la península ibérica. Annales Universitatis Scientiarium Budapestinensis de Rolando Eotvos Nominatae 25: I67-I84.

Coulmas, Florian. I979. On the sociolinguistic relevance of routine formulae. Journal of Pragmatics 3: 239-266. doi: https://doi.org/10.1016/0378-2166(79)90033-X

García Benito, Ana Belén. (2020). National Equities portuguesas e fraseologia para trabalhar conteúdos culturais nas aulas de PLE. En Silva, Suzete (org.) Fraseologia a Cia. Entabulando diálogos reflexivos.Campinas, São Paulo: Pontes Editores [2016]: 203-228.

Iriarte Sanromán, Álvaro. 200I. A Unidade Lexicográfica. Palavras, Colocações, Frasemas, Pragmatemas. Braga: Centro de Estudos Humanísticos-Universidade do Minho.

Luque Nadal, Lucía. 2008. Sobre los límites de la fraseología. Dichos y locuciones pragmático-conversacionales de carácter burlesco en español. Language Design I0: 87-106.

Martins, Vicente de Paula. 2020. Guia teórico para o estudo da fraseologia portuguesa. São Carlos: Pedro \& João Editores.

Mendivil-Giró, José Luis. I998. Aspectos teóricos del estudio de las unidades fraseológicas: gramática, pragmática y fraseología. En Wotjak, Gerd (ed) Estudios de fraseología y fraseografía del español actual. Frankfurt/Madrid: Vervuert/ Iberoamericana: 39-55.

Monteiro-Plantin, Rosemeire Selma. 20I4. Fraseologia: era uma vez um patinho feio no ensino de língua materna (volume I). Fortaleza: Imprensa Universitária.

Montoro del Arco, Esteban. 20I3. Valores pragmáticos de las locuciones pronominales. En Olza, Inés \& Manero, Elvira (ed.) Fraseopragmática. Berlin: Frank \& Timme GmbH Verlag für wissenschaftliche Literatur: 375-398.

Olza, Inés \& Manero, Elvira. 20I3. Introducción: el necesario "giro pragmático" en la fraseología. En Olza, Inés \& Manero, Elvira (ed.) Fraseopragmática. Berlin: Frank \& Timme GmbH Verlag für wissenschaftliche Literatur: 7-2.

Penadés Martínez, Inmaculada. I997. Aproximacion pragmatica a las unidades fraseologicas. En Escavy, R. et al. (eds.) Homenaje al profesor A. Roldan. Murcia: Universidad de Murcia: 4II-426.

Pereira, Antonio. 20I5. Competência linguística em português europeu língua materna e língua não materna: aquisição, ensino e aprendizagem das expressões idiomáticas. Tese de Doutoramento. Doutoramento em Ciências da Linguagem. Área de Conhecimento em Linguística Portuguesa. Universidade do Minho.

Quiroga Munguía, Paula. 2004. Enunciados fraseológicos: fórmulas rutinarias español/italiano. Lenguaje y textos 22: 23-33.

Sevilla Muñoz, Julia.; Zurdo Ruiz-Ayúcar, M. I. T. (dir.). 2009. Refranero multilingüe. Madrid. Instituto Cervantes (Centro Virtual Cervantes). http://cvc.cervantes. es/lengua/refranero/ [Acceso 20/09/2020] 
Solano Rodríguez, M. ${ }^{a}$ Ángeles. 2007. El papel de la conciencia fraseológica en la enseñanza y aprendizaje de una lengua extranjera. En González Rey, M. ${ }^{a}$ Isabel (coord.) Les expressions figées en didactique des langues étrangères. Louvain-La-Neuve (Bélgica): E.M.E (Éditions Modulaires Européennes)/ InterCommunications S.P.R.L.: 2OI-22I.

Solano Rodríguez, M. ${ }^{a}$ Ángeles. 20I2. Las unidades fraseológicas del francés y del español: tipología y clasificación. Paremia 21: II7-I28.

Solano Rodríguez, M. a Ángeles. 20I7. Enunciados fraseológicos pragmáticos. En Alessandro, Arianna; Solano Rodríguez, M. ${ }^{a}$ Ángeles; Zamora Muñoz, Pablo \& Sevilla Muñoz, Manuel. Introducción a la fraseología y la paremiología. Televisión universitaria de la UM. Universidad de Murcia. http://tv.um.es/video?i$\mathrm{d}=9039 \mathrm{I} \& \operatorname{cod}=\mathrm{aI}[$ Acceso IO/OI/2020] 
